\title{
Effectiveness of the 2010/11 seasonal trivalent influenza vaccine in Spain: preliminary results of a case-control study
}

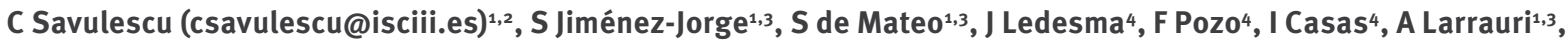
cycEVA Study Team ${ }^{5}$

1. National Centre of Epidemiology, Institute of Health Carlos III, Madrid, Spain

2. EpiConcept, Paris, France

3. Ciber Epidemiología y Salud Pública (CIBERESP), Ministry of Science and Innovation, Institute of Health Carlos III, Spain

4. National Influenza Centre-Madrid, National Centre for Microbiology, Institute of Health Carlos III, Madrid, Spain

5. Members of the team are listed at the end of the article

Citation style for this article:

Savulescu C, Jiménez-Jorge S, de Mateo S, Ledesma J, Pozo F, Casas I, Larrauri A, cycEVA Study Team. Effectiveness of the 2010/11 seasonal trivalent influenza vaccine in Spain: preliminary results of a case-control study.

Euro Surveill. 2011;16(11):pii=19820. Available online: http://www.eurosurveillance.org/ViewArticle.aspx?Articleld=19820

Article published on 17 March 2011

We present preliminary results of a case-control study to estimate influenza vaccine effectiveness in Spain, from week 50 of 2010 to week 6 of 2011. The adjusted effectiveness of the vaccine in preventing laboratoryconfirmed influenza due to any type of influenza virus was $50 \%$ (95\% Cl: -6 to $77 \%$ ) for the trivalent seasonal vaccine and $72 \%$ (95\% Cl: 7 to $92 \%$ ) for both trivalent seasonal and monovalent pandemic vaccines, suggesting a protective effect of seasonal vaccination lower than that reported for the previous season.

\section{Background}

After the 2009 influenza $\mathrm{A}\left(\mathrm{H}_{1} \mathrm{~N}_{1}\right)$ pandemic, the World Health Organization (WHO) in February 2010 recommended the trivalent influenza vaccine for the northern hemisphere for the $2010 / 11$ influenza season. The vaccine included the pandemic strain A/California/07/2009 (H1 subtype), the A/Perth/16/2009 (H3 subtype) and the $B /$ Brisbane $60 / 2008$ viruses. The influenza $A\left(\mathrm{H}_{1}\right)$ strain is the same as that used in the monovalent 2009/10 pandemic vaccine, which showed good effectiveness in preventing influenza $A\left(\mathrm{H}_{1} \mathrm{~N}_{1}\right)_{2009}$ infection in the $2009 / 10$ season $[1,2]$.

In Spain, influenza vaccination is offered free of charge each year to people in high-risk groups. In the 2010/11 season, it was recommended to persons over six months old with chronic conditions, elderly people aged over 60 years (65 years in some regions), healthcare workers and caregivers. The vaccination campaign lasted between September and November 2010 and several vaccine brands were used [3]. The monovalent pandemic vaccine was only offered in the 2009/10 season: the vaccine brands were mainly adjuvanted, except those used for pregnant women, for whom a non-adjuvanted vaccine was recommended. The pandemic vaccine was also not recommended for elderly people aged over 64 years without underlying diseases.
Since the 2008/09 influenza season, Spain has been participating in the Influenza Monitoring Vaccine Effectiveness in Europe (I-MOVE) network, established by the European Centre for Disease Prevention and Control (ECDC) [4]. Various study designs were tested: the test-negative case-control design proved suitable for such studies in Spain $[5,6]$. One of the objectives of this network is to provide early intraseasonal estimates of influenza vaccine effectiveness. The importance of having such estimates early in the season was highlighted during 2009/10, when intraseasonal estimates were needed in order to evaluate the impact of vaccination with the monovalent pandemic influenza vaccine [7].

The study presented here aims at providing an intraseasonal estimate of the seasonal trivalent vaccine 2010/11 effectiveness in preventing laboratory-confirmed influenza in Spain, in order to guide public health policies.

\section{Methods}

We conducted an observational case-control study (cycEVA) using the test-negative design described previously for the study of influenza vaccine effectiveness in elderly people [5]. Our study was carried out between week 50 of 2010 (12-18 December 2010) - when the influenza-like illness (ILI) threshold was first passed in the participating regions - and week 6 of 2011 (6-12 February 2011). Of the 17 regions of the Spanish Influenza Sentinel Surveillance System, eight participated in the study. In these eight regions, 246 of $325(76 \%)$ sentinel general practitioners (GPs) and paediatricians agreed to take part in the study, covering a population of 313,734 inhabitants, representing $2.1 \%$ of the total population in these regions [8]. Of the 246 GPs and paediatricians, $159(65 \%)$ recruited at least one patient in the study. 
Each week, participating GPs and paediatricians systematically swabbed the first two patients presenting with ILI according to the European Union case definition [8]. A case of confirmed influenza was defined as an ILI patient with laboratory confirmation of influenza virus infection. Three outcomes were used in the study: infection with any type of influenza virus, influenza $A\left(\mathrm{H}_{1} \mathrm{~N}_{1}\right)_{2009}$ virus and influenza $\mathrm{A}\left(\mathrm{H}_{3}\right)$ or influenza $B$ viruses. The controls were ILI patients whose laboratory results were negative for any influenza strain.

\section{Data collection}

Using a standardised questionnaire, participating GPs and paediatricians collected the following data for the recruited patients: age, sex, clinical symptoms, date of symptom onset, date of swabbing, vaccination status for 2010/11 seasonal influenza vaccine, influenza vaccination status for the previous season (seasonal and pandemic vaccines), laboratory result, chronic conditions, pregnancy, morbid obesity (defined as body mass index greater than 40), smoker status (current versus previous or non-smoker), functional status, any hospitalisation for chronic conditions in the previous year and the number of outpatient visits for any reason in the previous year. The patients were defined as having a chronic condition if they had any of the following: diabetes mellitus, cardiovascular disease, chronic pulmonary disease, renal disease, hepatic disease, congenital or acquired immunodeficiency, and chronic treatment with acetylsalicylic acid (in children). Poor functional status was defined as needing help for walking or bathing. Individuals were considered vaccinated if they had received the seasonal influenza

\section{FIGURE 1}

Laboratory-confirmed influenza cases $(n=629)$ and testnegative controls $(n=449)$ among ILI patients by week of swabbing, cycEVA study, week 50 (2010)-week 6 (2011) and weekly ILI incidence, week 40 (2010)-week 6 (2011), Spain

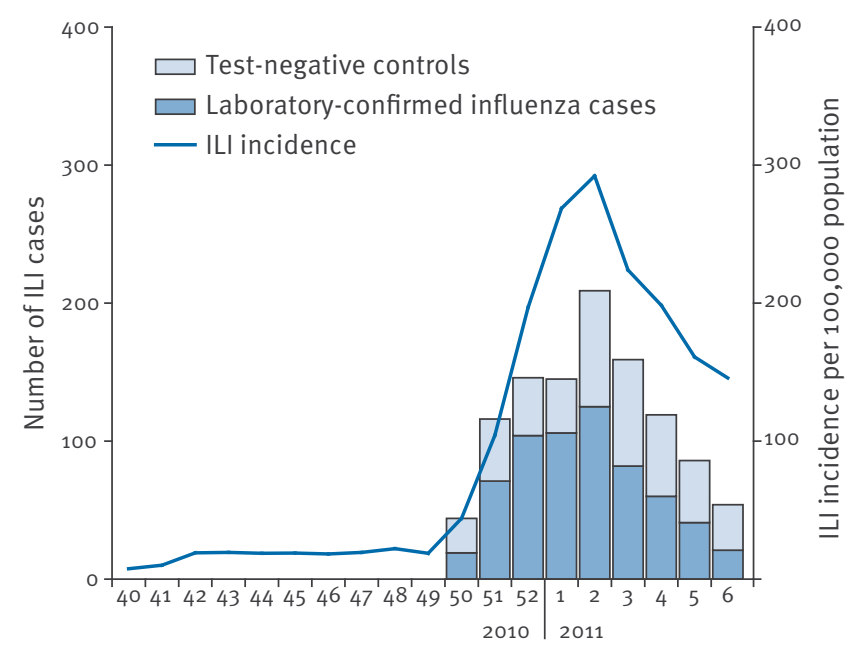

Week of swabbing

ILI: influenza-like illness.

Source: cycEVA study and Spanish Influenza Surveillance System, National Centre of Epidemiology, Institute of Health Carlos III, Spain. vaccine 14 days or more before the date of symptom onset. Vaccinated individuals whose date of vaccination was missing $(n=7)$ were considered vaccinated if the date of onset was two weeks after the end of the vaccination campaign.

\section{Data analysis}

We restricted all analyses to patients with an interval between symptom onset and swabbing of less than eight days. Logistic regression was used to calculate the crude and adjusted odds ratios (ORs) and their corresponding $95 \%$ Cls. Vaccine effectiveness was calculated as (1-OR) multiplied by 100 . All variables collected in the study were checked for possible confounding: we included in the regression model those that changed the crude OR by $>10 \%$. Thus, the final model included age group (0-4, 5-14, 15-44, 45-64 and $\geq 65$ years), week of swabbing and previous vaccination status (seasonal or pandemic vaccine, according to the analysis performed).

We first carried out the analysis with all eligible patients, as some previously healthy people might have been vaccinated in an occupational setting or in private clinics. Then we restricted the analysis to those eligible for vaccination (people in high-risk groups) [3]). To check the effect of being vaccinated with both vaccines when using influenza $A\left(\mathrm{H}_{1} \mathrm{~N}_{1}\right)_{2} 009$ virus infection as the outcome, we also carried out the analysis using a categorical variable for vaccination (unvaccinated, vaccinated with only seasonal trivalent vaccine 2010/11, only monovalent 2009/10 pandemic vaccine and both vaccines) [10]. We conducted all statistical analyses using STATA/IC 11.

The surveillance-affiliated laboratories or the National Centre of Microbiology (WHO National Influenza Centre-Madrid) confirmed influenza infection using real-time polymerase chain reaction (PCR). A number of laboratory-confirmed cases were genetically studied by sequencing the viral haemagglutinin gene. Phylogenetic analysis was carried out in order to characterise the specific strains of influenza $A$ and $B$ viruses.

The cycEVA study was included as part of influenza surveillance activities in Spain: therefore no ethical approval was needed for the study. No personal data were collected and patients gave verbal informed consent to be swabbed.

\section{Results}

From the beginning of the 2010/11 season in Spain, influenza $A\left(\mathrm{H}_{1} \mathrm{~N}_{1}\right) 2009$ virus has been predominant, with an increasing contribution of influenza B virus after the week 2 of 2011 when the peak of influenza activity was registered [11]. A similar viral circulation pattern and influenza activity evolution has been observed in the eight cycEVA regions. The incidence of ILI peaked in week 2 of 2011 (294 ILI cases per 100,000 population in the participating regions) (Figure 1). The 
highest incidence was recorded in children under 15 years, with a maximum weekly incidence of 543 and 533 ILI cases per 100,000 population in the age group 5-14 years and 0-4 years, respectively. During the study period, the proportion of influenza virus-positive samples increased from $40.3 \%$ in week 50 of 2010 to $64.3 \%$ in the epidemic peak and then decreased to $48.4 \%$ in week 06 of 2011 [11].

A total of 1,078 patients were recruited. Of these, 1,061 (98\%), comprising 618 cases and 443 controls, were included in the analysis where the outcome was laboratory confirmation of any type of influenza virus. For the analysis in which influenza $A\left(\mathrm{H}_{1} \mathrm{~N}_{1}\right)_{2009}$ infection was the outcome, we included 983 patients: 540 were laboratory-confirmed cases. When influenza $\mathrm{A}\left(\mathrm{H}_{3}\right)$ virus or influenza $B$ virus infection was the outcome, 513 patients were included: six were laboratory-confirmed cases of influenza $\mathrm{A}\left(\mathrm{H}_{3}\right)$ infection and 64 were laboratory-confirmed cases of influenza B infection (Figure 2).

The number of patients recruited in the study peaked in week 2 of 2011 and decreased thereafter during the study period, following the weekly ILI incidence in the eight participating regions (Figure 1).

Laboratory-confirmed influenza cases and test-negative controls did not differ regarding the covariates collected, except for age group and eligibility for vaccination (Table 1). Among cases, $53.9 \%$ belonged to the age group $15-44$ years compared with $47.6 \%$ of controls, and $3.6 \%$ of cases belonged to the age group $\geq 65$ years compared with $8.6 \%$ of controls. A higher proportion of patients were eligible for vaccination among controls (11.5\%) than among cases (7.9\%).

\section{FIGURE 2}

Flowchart of data exclusion and analysis outcomes, cycEVA study, Spain, week 50 (2010)-week 6 (2011)

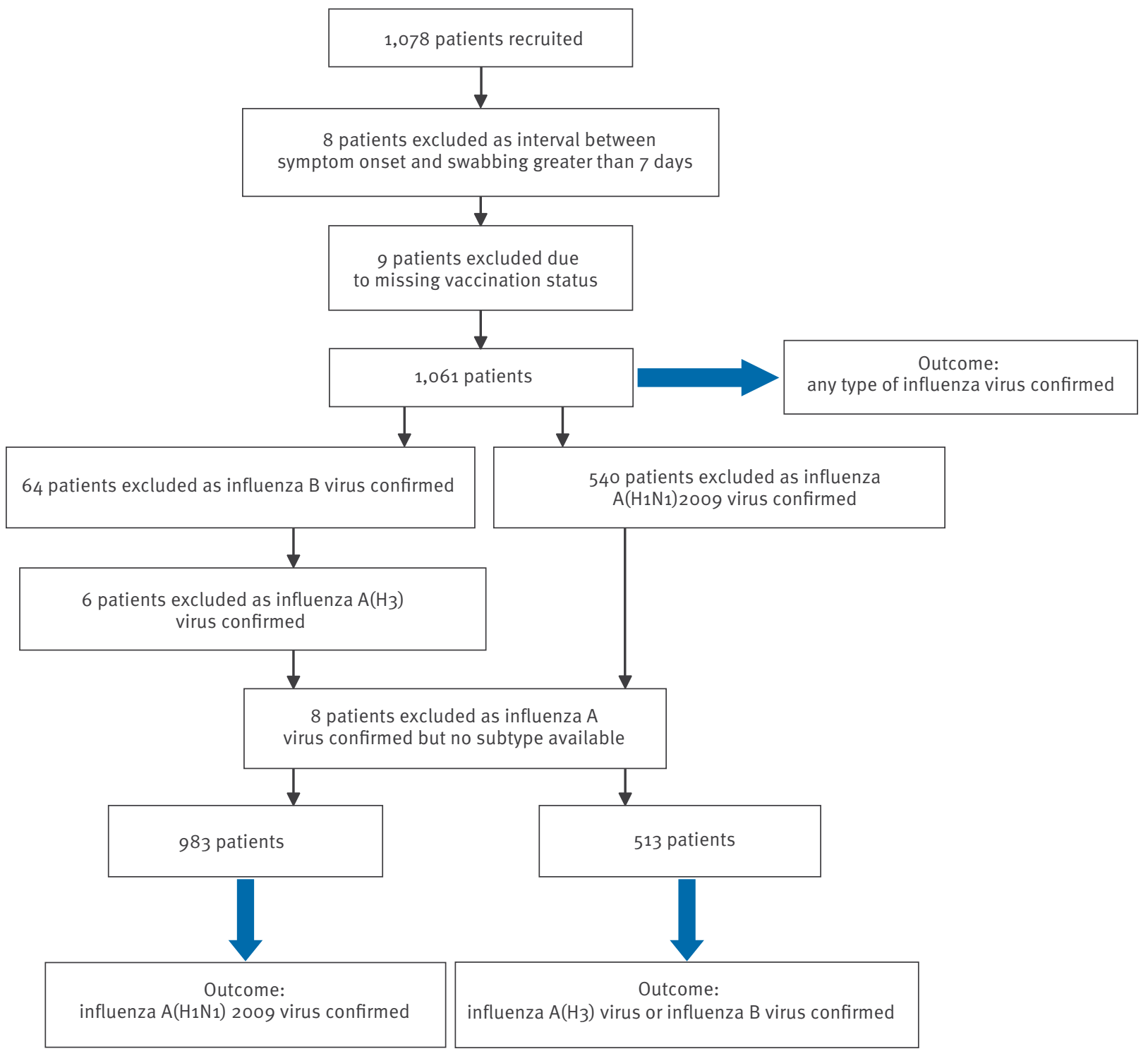


Estimates of the effectiveness of the seasonal

trivalent influenza vaccine $2010 / 2011$

The crude effectiveness of the vaccine in preventing influenza caused by any type of influenza virus was $65 \%$ (95\% Cl: 41-79\%). Adjusting for age group, monovalent pandemic vaccination, previous seasonal vaccination in 2009/10 and week of swabbing, the effectiveness was $50 \%$ (95\% Cl:-6 to $77 \%)$. In the

group eligible for vaccination ( $n=91)$, the adjusted vaccine effectiveness was $66 \%$ ( $95 \% \mathrm{Cl}:-1$ to $89 \%$ ).

In the analysis with influenza $A\left(\mathrm{H}_{1} \mathrm{~N}_{1}\right) 2009$ virus infection as the outcome, the crude vaccine effectiveness was $66 \%(95 \% \mathrm{Cl}: 41-81 \%)$ and the adjusted

\section{TABLE 1}

Characteristics of influenza cases with any type of influenza virus $(n=618)$ and test-negative controls $(n=443)$, cycEVA study, Spain, week 50 (2010)-week 6 (2011)

\begin{tabular}{|c|c|c|c|}
\hline Characteristic & $\begin{array}{c}\text { Cases }^{\mathrm{a}} \\
\text { No./total no. }(\%)^{\mathrm{b}}\end{array}$ & $\begin{array}{c}\text { Controls }^{\mathrm{a}} \\
\text { No./total no. }(\%)^{\mathrm{b}}\end{array}$ & Pvalue $^{c}$ \\
\hline \multicolumn{4}{|l|}{ Vaccination status } \\
\hline Vaccinated with trivalent $2010 / 11$ seasonal vaccine & $26 / 618(4.2)$ & 49/443(11.1) & $<0.0001$ \\
\hline Vaccinated with monovalent $2009 / 10$ pandemic vaccine & $12 / 594(2.0)$ & $24 / 398(6.0)$ & 0.001 \\
\hline \multicolumn{4}{|l|}{ Age group (years) } \\
\hline $0-4$ & $44 / 618(7.1)$ & $32 / 443(7.2)$ & \multirow[t]{5}{*}{0.007} \\
\hline $5-14$ & $101 / 618(16.3)$ & $80 / 443(18.1)$ & \\
\hline $15-44$ & $332 / 618(53.9)$ & $211 / 443(47.6)$ & \\
\hline $45-64$ & $118 / 618(19.1)$ & $82 / 443(18.5)$ & \\
\hline$\geq 65$ & $22 / 618(3.6)$ & $38 / 443(8.6)$ & \\
\hline Male & $300 / 618(48.6)$ & $204 / 443(46.0)$ & 0.422 \\
\hline Any chronic condition & $67 / 450(14.9)$ & $61 / 330(18.5)$ & 0.180 \\
\hline Pregnancy & $1 / 255(0.4)$ & $5 / 217(2.3)$ & 0.065 \\
\hline Obesity $^{d}$ & 4/475 (0.8) & $3 / 349$ (0.9) & 0.978 \\
\hline Any hospitalisation for chronic conditions in the previous year & 4/611 (0.6) & $8 / 431(1.9)$ & 0.073 \\
\hline \multicolumn{4}{|l|}{ Number of visits to a GP in the previous year } \\
\hline None & $164 / 610(26.9)$ & $96 / 432(22.2)$ & \multirow[t]{3}{*}{0.107} \\
\hline $1-4$ & $256 / 610(42.0)$ & $178 / 432(41.2)$ & \\
\hline$>4$ & $190 / 610(31.2)$ & $158 / 432(36.6)$ & \\
\hline Smoking & $47 / 532(8.8)$ & $38 / 366(10.4)$ & 0.436 \\
\hline Poor functional status & $2 / 571(0.3)$ & 4/393 (1.0) & 0.195 \\
\hline Eligible for vaccination & 49/618 (7.9) & $51 / 443(11.5)$ & 0.049 \\
\hline
\end{tabular}

GP: general practitioner.

a Cases and controls recruited with an interval between symptom onset and swabbing of less than eight days.

${ }^{b}$ Unless otherwise indicated.

'Chi-square test or Fisher's exact test, when appropriate.

${ }^{d}$ Defined as body mass index greater than 40.

\section{TABLE 2}

Intraseasonal estimates of trivalent 2010/11 seasonal influenza vaccine and monovalent 2009/10 pandemic vaccine in preventing influenza A(H1N1) 2009 infection, Spain, week 50 (2010)-week 6 (2011)

\begin{tabular}{|c|c|c|c|c|c|}
\hline Patients & Vaccination status & $\begin{array}{l}\text { Number } \\
\text { of cases }\end{array}$ & $\begin{array}{l}\text { Number of } \\
\text { controls }\end{array}$ & $\begin{array}{c}\text { Crude vaccine effectiveness, } \\
\text { as percentage } \\
(95 \% \mathrm{Cl})\end{array}$ & $\begin{array}{c}\text { Adjusted vaccine effectiveness }{ }^{\mathrm{a}} \text {, } \\
\text { as percentage } \\
(95 \% \mathrm{Cl})\end{array}$ \\
\hline \multirow{4}{*}{ All $^{\mathrm{b}}$} & Unvaccinated & 494 & 344 & Reference & Reference \\
\hline & Seasonal $2010 / 11$ vaccine only & 18 & 30 & $58(24$ to 77$)$ & $52(6$ to 75$)$ \\
\hline & Pandemic $2009 / 10$ vaccine only & 5 & 9 & $61(-16$ to 87$)$ & $67(-5$ to 90$)$ \\
\hline & Seasonal and pandemic vaccines & 4 & 15 & $82(44$ to 94$)$ & 72 (7 to 92) \\
\hline \multirow{4}{*}{$\begin{array}{l}\text { Eligible for } \\
\text { vaccination }^{c}\end{array}$} & Unvaccinated & 27 & 20 & Reference & Reference \\
\hline & Seasonal $2010 / 11$ vaccine only & 9 & 17 & $61(-6$ to 86$)$ & $52(-53$ to 85$)$ \\
\hline & Pandemic $2009 / 10$ vaccine only & 2 & 0 & ND & ND \\
\hline & Seasonal and pandemic vaccines & 3 & 10 & 78 (9 to 95) & 83 (15 to 97$)$ \\
\hline
\end{tabular}

$\mathrm{Cl}$ : confidence interval; ND: not determined.

${ }^{a}$ Adjusted for age group and week of swabbing.

${ }^{b}$ Includes 521 cases and 398 controls.

c Includes 41 cases and 47 controls. 
effectiveness estimate, taking into account age group, monovalent pandemic vaccination and week of swabbing, was $49 \%$ (95\% Cl: $3-73 \%)$. For those eligible for seasonal vaccination $(n=88)$, the adjusted vaccine effectiveness was $63 \%$ ( $95 \% \mathrm{Cl}:-15$ to $88 \%$ ).

Crude vaccine effectiveness in preventing influenza $\mathrm{A}\left(\mathrm{H}_{3}\right)$ virus or influenza $\mathrm{B}$ virus infection was $51 \%(95 \%$ $\mathrm{Cl}:-40$ to $88 \%$ ), which increased when adjusted for age group, previous seasonal vaccination in 2009/10 and week of swabbing to $84 \%$ (95\% Cl:16-97\%). For those eligible for vaccination, the adjusted vaccine effectiveness was $90 \%$ ( $95 \% \mathrm{Cl}$ : -80 to $100 \%$ ).

In the analysis with the four-level vaccination variable in preventing influenza $A\left(\mathrm{H}_{1} \mathrm{~N}_{1}\right)_{2009}$ infection, in patients who received $2010 / 11$ seasonal trivalent vaccine only, the vaccine effectiveness, adjusted for age group and week of swabbing, was 52\% (95\% Cl: 6-75\%) (Table 2). For patients receiving both seasonal trivalent and monovalent pandemic vaccines, the adjusted vaccine effectiveness was $72 \%$ (95\% Cl: $7-92 \%)$. In the analysis including patients eligible for vaccination, the adjusted effectiveness when vaccinated with both vaccines was ( $83 \% ; 95 \% \mathrm{Cl}: 15-97 \%)$. Point estimates for patients vaccinated only with the pandemic vaccine were higher than for the patients vaccinated only with the $2010 / 11$ seasonal vaccine, but the difference was not statistically significant (Table 2 ).

\section{Laboratory findings}

A total of 56 specimens were sent for genetic characterisation of the virus. In 40 specimens, there was sufficient PCR-amplified product for sequencing of the viral haemagglutinin gene: 33 were influenza $A\left(H_{1} N_{1}\right)_{2009}$, one was influenza $A\left(H_{3}\right)$ and six were influenza $B$ viruses. Phylogenetic analysis of the 33 $A\left(\mathrm{H}_{1} \mathrm{~N}_{1}\right)_{2009}$ sequences showed a genetic similarity to the influenza virus of the pandemic vaccine since neither specific mutations $94 \mathrm{~N}, 125 \mathrm{D}$ and $250 \mathrm{~A}$ defining the $A / C h r i s t c h u r c h / 16 / 2010$ clade, nor $128 \mathrm{P}, 199 \mathrm{~A}$ and $295 \mathrm{~V}$ defining the $\mathrm{A} / \mathrm{Hong} \mathrm{Kong} / 2213 / 2010$ clade were found. Nevertheless, three of the 33 sequenced viruses showed other amino acid changes compared with the vaccine strain. The six influenza $B$ viruses were similar to the vaccine strain. Specific mutations $53 \mathrm{~N}, 94 \mathrm{H}, 230 \mathrm{~V}$ and $280 \mathrm{~A}$, defining the clade A/Hong Kong 2121/2010 were identified for the patient with influenza $\mathrm{A}\left(\mathrm{H}_{3}\right)$ virus.

\section{Discussion}

Our results suggest a protective effect of the seasonal trivalent vaccine in preventing influenza due to infection of any type of influenza virus, including influenza $A\left(\mathrm{H}_{1} \mathrm{~N}_{1}\right)_{2009}$ virus and influenza $A\left(\mathrm{H}_{3}\right)$ or influenza $B$ viruses. Similar results were obtained when we restricted the analysis to those eligible for vaccination. These are preliminary results and should be interpreted with caution, taking into consideration the sample size.
However, the effectiveness of the trivalent seasonal vaccine in preventing influenza $\mathrm{A}\left(\mathrm{H}_{1} \mathrm{~N}_{1}\right)_{2009}$ infection in both analyses ( $49 \%$ and $52 \%$ ) is lower than that reported for the monovalent pandemic vaccine in the $2009 / 10$ season in the same study population, which reached $75 \%$ (unpublished data). Several factors might have contributed to this finding. Firstly, the monovalent pandemic vaccine used in the $2009 / 10$ season was adjuvanted (with the exception of that used for pregnant women), while the current seasonal trivalent vaccine used in all participating regions is non-adjuvanted. Secondly, the monovalent pandemic vaccine was not recommended for elderly people aged over 64 years without underlying diseases, resulting in a vaccinated population that was younger and more immunocompetent. Last, but not least, the lower effectiveness of the seasonal vaccine might suggest that there may have been some genetic changes in the influenza $A\left(\mathrm{H}_{1} \mathrm{~N}_{1}\right) 2009$ virus. Most influenza $A\left(\mathrm{H}_{1}\right)$ viruses circulating in Spain remained closely related genetically to the vaccine virus; however, there have been observed some amino acid changes in the haemagglutinin gene of a small proportion of studied strains that could be reasonably be attributable to genetic drift, since these mutations are different from those defining new clades observed in September 2010 [12]. Notably, the only influenza $\mathrm{A}\left(\mathrm{H}_{3}\right)$ virus characterised in our study falls within a subgroup represented by the influenza A/ Hong Kong/2121/2010 virus.

We also observed a higher protective effect in preventing infection due to influenza $A\left(\mathrm{H}_{1} \mathrm{~N}_{1}\right) 2009$ virus in patients who had received both seasonal trivalent and monovalent pandemic vaccines, consistent with other early reports $[10,13]$.This might suggest a type of cumulative protection, which should be confirmed by immunological studies, and highlights the need for routine annual influenza vaccination for people in the recommended groups.

In the same analysis, we also found that the monovalent pandemic vaccine had a higher point estimate than that for the seasonal vaccine, but this difference was not statistically significant due to the low number who were vaccinated. These findings might be related again to the type of the vaccine used (adjuvanted versus non-adjuvanted) or to the population targeted for vaccination.

Interestingly, we found a good protective effect of the seasonal trivalent vaccine against influenza $\mathrm{A}\left(\mathrm{H}_{3}\right)$ and influenza B viruses, although this effect was higher than that reported in another study [10]. This is consistent with the good match between the vaccine and circulating influenza B strain. The difference in the estimates could be related to different confounding factors that the effectiveness calculations were adjusted for.

This is the third season in which we have used the test-negative case-control design in the cycEVA study. The experience of the two previous seasons $[1,5]$ was 
reflected in increased participation of GPs and paediatricians, compliance with the protocol and completeness of data collection (less than 10\% data were missing for important variables). The introduction of systematic swabbing for ILI patients might have reduced the selection bias toward vaccinated patients, which is known to occur in surveillance-based studies [14].

In conclusion, the cycEVA study was able to provide an early intraseasonal estimate of the effectiveness of the seasonal vaccine nine weeks since the epidemic started. It suggests a protective effect of the vaccine against all types of influenza viruses. This effect was also seen in the group eligible for vaccination; however, the effect was lower than that reported in the previous season [1]. It also demonstrates that intraseasonal vaccine effectiveness estimates are possible by conducting observational studies, with an acceptable additional effort, within the framework of a well-organized influenza surveillance system meeting the criteria of the European Influenza Surveillance Network.

The cycEVA study is ongoing in Spain and ILI cases are still being recruited while sporadic circulation of influenza viruses is registered in the participating regions. Therefore we expect that at the end of the season the sample size will allow more precise estimates of vaccine effectiveness and will enable us to control for other confounding factors known to influence vaccine effectiveness. In addition, the I-MOVE multicentre study, pooling data from eight European countries including Spain, will be able to present even more precise estimates.

\section{Acknowledgements}

We are grateful to sentinel GPs, paediatrician and virologist participating in the cycEVA study, as well as to all professionals participating in the Spanish Influenza Surveillance System. We thank Isabel Pachón y Aurora Limia (Coordinating Centre for Health Alerts and Emergencies within Spanish Ministry of Health and Social Policy) for the information provided on influenza vaccination. We also thank Marta Valenciano, Alain Moren (EpiConcept), and Bruno Ciancio (ECDC) for their support in the cycEVA study.

The cycEVA study was funded by ECDC through the I-MOVE project. The Institute of Health Carlos III also provided a research grant through the Influenza $\mathrm{A}\left(\mathrm{H}_{1} \mathrm{~N}_{1}\right)_{2009}$ Programme (GRog/0017).

\section{Members of the cycEVA Study Team}

Jaume Giménez, Juana M. Vanrell (Service of Epidemiology, General Directorate of Public Health, Baleares), Carolina Rodriguez, Tomás Vega (General Directorate of Public Health, Research and Development. Castilla y León), Ana Martínez, Nuria Torner (Department of Health, Generalitat of Catalonia. CIBERESP), Julián M. Ramos, Maria C. Serrano (Sub-directorate of Epidemiology, Public Health Directorate. Extremadura), Jesús Castilla, Manue García Cenoz (Institute of Public Health of Navarra. CIBERESP) Jone M. Altzíbar (Sub-directorate of Public Health. País Vasco. CIBERESP), Jose M. Arteagoitia (Public Health Service, Department of Health, Basque Government. País Vasco), Carmen Quiñones, Milagros Perucha (General Directorate of Public Health and Consumption, Consejería de Salud. La Rioja), Daniel Castrillejo (General Directorate of Public Health and Consumption of Melilla).

\section{References}

1. Valenciano M, Kissling E, Cohen J-M, Oroszi B, Barret A-S, Rizzo C, et al. Estimates of pandemic influenza vaccine effectiveness in Europe, 2009-2010: results of Influenza Monitoring Vaccine Effectiveness in Europe (I-MOVE) multicentre case-control study. PLoS Med. 2011;8(1):e1000388.

2. Hardelid P, Fleming DM, McMenamin J, Andrews N, Robertson C, SebastianPillai P, et al. Effectiveness of pandemic and seasonal influenza vaccine in preventing pandemic influenza $A$ $\left(\mathrm{H}_{1} \mathrm{~N}_{1}\right)_{2009}$ infection in England and Scotland 2009-2010. Euro Surveill. 2011;16(2):pii=19763. Available from: http://www. eurosurveillance.org/ViewArticle. aspx?Articleld=19763

3. Ministry of Health and Social Policy, Spain. Prevención de la gripe. Recomendaciones de vacunación antigripal [Influenza prevention. Recommendations for influenza vaccination]. Ministry of Health and Social Policy. [Accessed 28 Feb 2011]. Spanish. Available from: http://www.msps.es/ciudadanos/ enfLesiones/enfTransmisibles/gripe/home.htm

4. Valenciano M, Ciancio B, Moren A; Influenza Vaccine Effectiveness Working Group. First steps in the design of a system to monitor vaccine effectiveness during seasonal and pandemic influenza in EU/EEA Member States. Euro Surveill. 2008;13(43):pii=19015. Available from: http://www. eurosurveillance.org/ViewArticle.aspx?Articleld=19015

5. Savulescu C, Valenciano M, de Mateo S, Larrauri A; cycEVA Study Team. Estimating the influenza vaccine effectiveness in elderly on a yearly basis using the Spanish influenza surveillance network--pilot case control studies using different control groups, 2008-2009 season, Spain. Vaccine. 2010;28(16):2903-7.

6. Larrauri A, Savulescu C, Jiménez-Jorge S, Pérez-Breña P, Pozo F, Casas I, et al. Influenza pandemic ( $\left.\mathrm{H}_{1} \mathrm{~N}_{1}\right) 2009$ activity during summer 2009. Effectiveness of the 2008-9 trivalent vaccine against pandemic influenza in Spain. Gac Sanit. 2011;25(1):23-28.

7. European Centre for Disease Prevention and Control (ECDC). The $2009 \mathrm{~A}\left(\mathrm{H}_{1} \mathrm{~N}_{1}\right)$ pandemic in Europe. Stockholm: ECDC; 2010. Available from: http://www.ecdc.europa.eu/en/publications/ Publications/101108_SPR_pandemic_experience.pdf

8. Spanish National Institute of Statistic. Censo de Población y Viviendas 2001. [Census of Population and Housing 2001]. Spanish. Available from: http://www.ine.es/inebmenu/mnu_ cifraspob.htm

9. European Commission. Commission Decision of 30 April 2009 amending Decision 2002/253/EC laying down case definitions for reporting communicable diseases to the Community network under Decision No 2119/98/EC of the European Parliament and of the Council, Luxembourg: Publications Office of the European Union. 1.5.2009. L 110/58. Available from: http://eur-lex.europa.eu/LexUriServ/LexUriServ.do?uri= OJ:L:2009:110:0058:0059:EN:PDF

10. Pebody R, Hardelid P, Fleming DM, McMenamin J, Andrews N, Robertson C, et al. Effectiveness of seasonal 2010/11 and pandemic influenza $A\left(\mathrm{H}_{1} \mathrm{~N}_{1}\right)_{200} 20$ vaccines in preventing influenza infection in the United Kingdom: mid-season analysis 2010/11. Euro Surveill. 2011;16(6): pii=19791. Available from: http://www.eurosurveillance.org/ViewArticle. aspx?Articleld $=19791$

11. Instituto de Salud Carlos III. Vigilancia de la gripe en España. Informe semanal de vigilancia de gripe. Semana 06/2011 [Weekly report of the Spanish Influenza Surveillance System. Week 06/2011]. Spanish. Available from: http://vgripe.isciii.es/ gripe/documentos/20102011/boletines/grno62011.pdf

12. European Centre for Disease Prevention and Control (ECDC). Surveillance report. Community Network of Reference Laboratories (CNRL) for Human Influenza in Europe. Influenza virus characterisation. Summary for Europe, September 2010. Stockholm: ECDC. Available from: http://ecdc.europa. eu/en/publications/Publications/1010_Influenza_virus characterisation_2010_September.pd $\bar{f}$

13. Castilla J, Morán J, Martínez-Artola V, Reina G, Martínez-Baz I, García Cenoz M, et al. Effectiveness of trivalent seasonal and monovalent influenza $A\left(\mathrm{H}_{1} \mathrm{~N}_{1}\right)_{2} 009$ vaccines in population with major chronic conditions of Navarre, Spain: 2010/11 mid-season analysis. Euro Surveill, 2011:16(7): pii=19799. Available from: http://www.eurosurveillance.org/ViewArticle. aspx?Articleld $=19799$

14. Larrauri A, De Mateo S; Spanish Influenza Sentinel Surveillance System. Characterisation of swabbing for virological analysis in the Spanish Influenza Sentinel Surveillance System during four influenza seasons in the period 2002-2006. Euro Surveill. 2007;12(5):pii=706. Available from: http://www.eurosurveillance.org/ViewArticle. aspx?Articleld $=706$ 